OPEN ACCESS

Edited by:

Claire Perks,

University of Bristol, United Kingdom

Reviewed by:

Marcello Maggiolini,

University of Calabria, Italy

Silvère Baron,

U1103 Génétique Reproduction et Développement (GReD)(INSERM),

France

*Correspondence:

Gabriella Castoria

gabriella.castoria@unicampania.it

Antimo Migliaccio

antimo.migliaccio@unicampania.it

Specialty section: This article was submitted to

Cancer Endocrinology,

a section of the journal

Frontiers in Endocrinology

Received: 21 December 2021

Accepted: 20 January 2022

Published: 11 February 2022

Citation:

Licitra F, Giovannelli P, Di Donato $M$ Monaco A, Galasso G, Migliaccio A and Castoria G (2022) New Insights and Emerging Therapeutic Approaches in Prostate Cancer.

Front. Endocrinol. 13:840787. doi: 10.3389/fendo.2022.840787

\section{New Insights and Emerging Therapeutic Approaches in Prostate Cancer}

\author{
Fabrizio Licitra, Pia Giovannelli, Marzia Di Donato, Alessandra Monaco, \\ Giovanni Galasso, Antimo Migliaccio * and Gabriella Castoria* \\ Department of Precision Medicine, University of Campania 'L. Vanvitelli', Naples, Italy
}

Prostate cancer is the second most frequently diagnosed cancer in men and several therapeutic approaches are currently available for patient's care. Although the androgen receptor status represents a good predictor of response to androgen deprivation therapy, prostate cancer frequently becomes resistant to this approach and spreads. The molecular mechanisms that contribute to progression and drug-resistance of this cancer remain still debated. However, few therapeutic options are available for patient's management, at this stage. Recent years have seen a great expansion of the studies concerning the role of stromal-epithelial interactions and tumor microenvironment in prostate cancer progression. The findings so far collected have provided new insights into diagnostic and clinical management of prostate cancer patients. Further, new fascinating aspects concerning the intersection of the androgen receptor with survival factors as well as calcium channels have been reported in cultured prostate cancer cells and mouse models. The results of these researches have opened the way for a better understanding of the basic mechanisms involved in prostate cancer invasion and drugresistance. They have also significantly expanded the list of new biomarkers and druggable targets in prostate cancer. The primary aim of this manuscript is to provide an update of these issues, together with their translational aspects. Exploiting the power of novel promising therapeutics would increase the success rate in the diagnostic path and clinical management of patients with advanced disease.

\footnotetext{
Keywords: nerve growth factor signalling, calcium influx, cancer-associated fibroblasts, prostate cancer, new drugs

Abbreviations: $\alpha$-SMA, $\alpha$-smooth muscle actin; ADT, androgen deprivation therapy; AR, androgen receptor; $\mathrm{B} 2 \mathrm{R}$, bradykinin 2 receptor; BDNF, brain-derived neurotrophic factor; $\mathrm{BPH}$, benign prostatic hyperplasia; $\mathrm{CAF}$, cancer associated fibroblast; CHRM4, cholinergic receptor muscarinic 4; CRPC, castration resistant prostate cancer; CTD, C-terminal domain; EMT, epithelial-to-mesenchymal transition; FAP, fibroblast activating protein; FlnA, filamin A; HER3, Receptor tyrosine-protein kinase erbB-3; HIF- $1 \alpha$, hypoxia-induced factor $1 \alpha$; IFN- $\gamma$, interferon- $\gamma$; IHC, immunohistochemistry; M-CSF, macrophage colony stimulating factor; MAPK, mitogen-activated protein kinase; MHR, melastatin homology region; MMP, matrix metalloproteinase; NEPC, neuroendocrine prostate cancer; NGF, nerve growth factor; NGFR, nerve growth factor receptor; NGS, next generation sequencing; NRG1, neuregulin-1; NT, neurotrophin; PC, prostate cancer; PGC1 $\alpha$, peroxisome proliferator-activated receptor gamma coactivator 1- $\alpha$; PI3-K, phosphatidylinositol 3-kinase; PIN, prostatic intraepithelial neoplasia; PLC $\gamma$, phospholipase C $\gamma$; PSA, prostate specific antigen; RTK, receptor tyrosine-kinase; SIRT1, sirtuin 1; TGF- $\beta$, transforming growth factor- $\beta$; TMD, transmembrane domain; TME, tumor microenvironment; TrkA/B/C, tropomyosin receptor kinase A/B/C; TRPM8, transient receptor potential melastatin-8; VEGF-A, vascular endothelial growth factor-A.
} 


\section{INTRODUCTION}

Prostate cancer (PC) still remains the second most commonly diagnosed neoplasia in men (1). Depending on the availability of specific screenings, including the prostate-specific antigen (PSA) assay, the lifestyle and environmental factors, PC incidence varies among men of different ethnicities (2). Despite the recent advances in early diagnosis and detection, the disease's onset is often asymptomatic, accounting for numerous late diagnoses. Additionally, the prognosis can be favorable at early stage's disease, given the progresses of advanced radiotherapy technology (3, 4 and refs therein). However, PC still represents the second leading cause of cancer-related death in men, albeit its mortality rate is relatively low (almost 20-30\%), as compared with other solid cancers (5). New therapeutic options are needed for patients with advanced disease.

PC pathogenesis and progression depend on androgen/ androgen-receptor (AR) circuit. As such, the mainstream pharmacological approach relies on the androgen deprivation therapy (ADT), which shows a satisfactory response in a significant number of cases. However, many PC relapse and progress towards a more aggressive phenotype, often characterized by ADT insensitivity and androgenindependence. Such phenotype, also called castrate-resistant prostate cancer (CRPC), may be metastatic or not $(6,7)$. Additionally, a subset of PC might further differentiate into neuroendocrine phenotype, also called neuroendocrine PCs (NEPCs). These cancers lose AR signaling, become more aggressive and exhibit androgen-independence in a quite scantly known molecular landscape (8). Among the various factors elsewhere excellently discussed (9-11), PC progression is often characterized by abnormal AR-mediated signaling activation or AR variants (12), which might help the tumor to achieve ADT unresponsiveness. However, emerging findings have identified unexpected drivers of PC progression. Some of them are implied in the survival response elicited by the receptor tyrosine-kinase (RTK) signaling, such as the nerve growth factor (NGF) and its high-affinity receptor, tropomyosin-related kinase receptor A (TrkA; 13). Recent papers, including ours, have investigated this issue in PC (14-18). Other findings have identified the transient receptor potential melastatin-8 (TRPM8 ) as a playmaker in PC (19-21 and refs therein), likely because of its role in connecting the androgen endocrine system with intracellular calcium levels (22). At last, the role of cancerassociated fibroblasts (CAFs) in PC progression is undeniable (23). The finding that CAFs harbor significant amounts of AR has opened new ways for a better understanding of the role of tumor microenvironment in PC progression and more tailored approaches of this cancer (23-29).

In search for a link between these three apparently unlinked items, it might be argued that NGF and other neurotrophins secreted from PC cells or CAFs sustain tumor survival and aggressiveness through a paracrine loop, as it occurs in breast cancer (30-33). However, NGF and calcium signaling might intersect each other in PC, as it occurs in neurons (34) or Schwann cells (35). In this manuscript, we will discuss these emerging findings and their connections. The potential application of these data in diagnostic and therapeutic guidance of PC will be outlined.

\section{NERVE GROWTH FACTOR AND ITS RECEPTORS IN PROSTATE CANCER}

The action of neurotrophins is mediated by the binding to membrane receptors, mainly the neurotrophin receptor p75NTR (also called NGF receptor; NGFR) and the neurotrophin tyrosine kinase receptor (Trk) family, which consists of three members, TrkA, B and C, with a variable affinity for the four identified neurotrophins (NGF; brainderived neurotrophic factor, BDNF; neurotrophin 3 and 4, NT3 and NT4; Figure 1). Their dependent signaling mediates, indeed, the activation of several downstream effectors, such as MAPK, PI3-K, PLC $\gamma$ as well as the small GTP binding proteins that strongly impact differentiation and survival, cytoskeletal remodeling, receptor cross-talk and ion channels in various cell types, other than neurons (13). Beyond their role in neuronal cells, neurotrophic factors are emerging as potential drivers of cancer progression, and therapies specifically affecting the neurotrophin-mediated signaling might hold value for innovative treatments of human cancers $(36,37)$.

PCs release NGF and express the neurotrophin receptors, which undergo significant changes during PC progression, as primary PC express both TrkA and NGFR, while losing NGFR during the disease progression. This behavior has been linked with PC onset and androgen-resistance development (38). At last, NGFR is almost completely absent in metastatic PC, making the TrkA receptor the lead driver of NGF signaling in aggressive PC (16). Previous findings have reported that NGF triggers mitogenesis and promotes PSA release through TrkA activation in LNCaP cells, and this effect is additive to that exerted by androgens (39). As such, it was thereafter found that the tyrosine kinase inhibitor, CEP-701 blocks the TrkA-mediated events, thereby reducing invasiveness of PC cultured cells (40). Derangements of NGF and its dependent signaling can be often detected in PC (41), where the neurotrophin might be released by PC cells and/or the surrounding stromal cells. As such, a paracrine loop between the two counterparts occurs (42). We recently reported that a reciprocal cross-talk between $A R$ and TrkA fosters the mitogenesis or motility of LNCaP cells in response to NGF or androgens (15). The obvious impact of these findings is that combinatorial treatment with antiandrogens and TrkA inhibitors might be explored in PC patients. Consistent with the hypothesis that TrkA is involved in PC motility and spreading, it has been shown that nonproteolytic ubiquitination of TrkA by the ubiquitin-ligase TRAF4 increases the kinase activity of the receptor and mediates PC spreading. The finding that TRAF4 is overexpressed in advanced PC specimens strongly supports the involvement of this mechanism in PC aggressiveness (14). The role of NGF in PC malignancy has been further highlighted by the finding that NGF-elicited activation of TrkA increases mitogenesis, epithelial-mesenchyme transition (EMT) and 


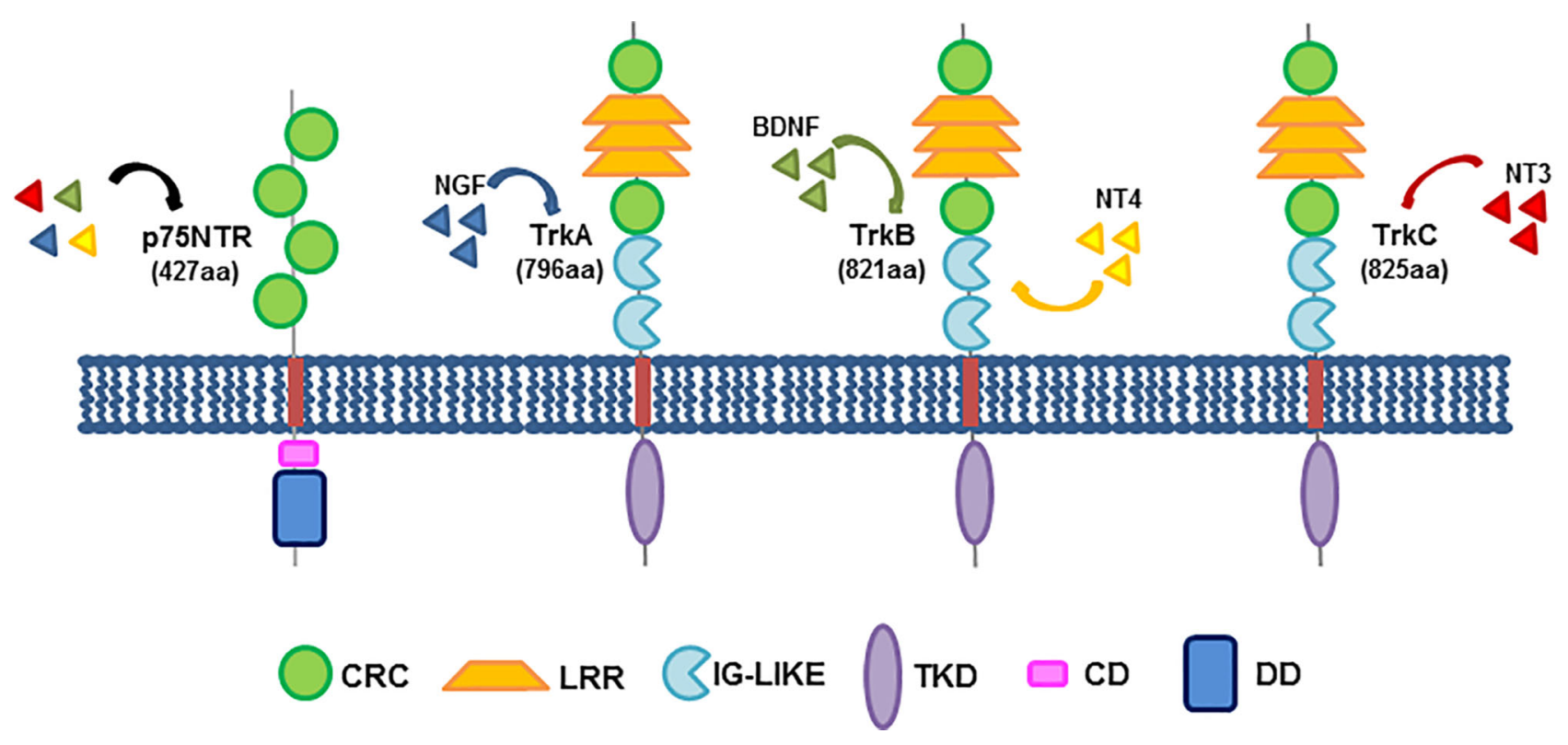

FIGURE 1 | Neurotrophin receptors and their structure. The three members of the Trk family share a common structure, encompassing a cysteine-rich cluster $(\mathrm{CRC})$, three leucine-rich repeats (LRR), a second cysteine cluster and two immunoglobulin-like domains (IG-Like), responsible for ligand binding, in their extracellular domains. A transmembrane domain (TMD) anchors the receptor to the plasma membrane. The intracellular region consists of the tyrosine kinase domain (TKD). On the left, the low-affinity neurotrophin receptor, p75NTR (called NGFR) is also depicted. It consists of four cysteine-rich clusters in the extracellular domain, a transmembrane region and an intracellular region exhibiting a chopper domain (CD) and a death domain (DD). Shown beside each Trk member is reported the corresponding high-affinity binding neurotrophin. p75NTR might bind all neurotrophins at low affinity. The indicated domains are colored as follows: CRC, green; LRR, yellow; IG-Like, light blue; TMD, red; TKD, purple; CD, pink; DD, dark blue.

invasion of various CRPC-derived cells through activation of the downstream Ras- and PI3-K-dependent signaling cascades. Chemical inhibitors of TrkA or siRNA approaches have definitely indicated a role for this receptor in NGF-elicited responses of CRPC-derived cells or spheroids (16). In addition to suggesting the clinical benefit from TrkA inhibitor usage in PC patients (37), the findings so far presented point to the role of NGF axis in PC survival. This circuit might substitute the androgens in controlling PC cell survival and lead to disease's progression towards the CRPC stage. As before stated, similar findings have been reported in various 'hormone-dependent' cancers, including breast cancers. Thus NGF might intersect the steroid endocrine system in various solid cancers.

Beyond the well described mechanism(s) responsible for PC progression and drug-resistance, neuroendocrine differentiation of PC is recently emerging as a process by which a subset of CRPC escapes the ADT. These tumors acquire some signatures (low or absent AR signaling, $\mathrm{Rb}$ and p53 loss, amplification of Myc-N and epigenetic changes) which lead to a highly aggressive phenotype and patient's death within 2 years ( 8 and therein refs). To date, no therapies are available for NEPC patients. Therefore, the identification of NEPC drivers represents a major challenge. Some years ago, it was shown that PC cells overexpress Myc-N after a prolonged ADT. The oncogene activation correlated with a low or absent AR expression. It was proposed that this feature leads to development of undifferentiated, invasive PC cells that exhibit characteristics similar to those of human NEPC (43).
Subsequent reports have confirmed that a small fraction of PCs differentiate into NEPCs upon protracted ADT. These cancers lose the AR-dependent signaling and progress towards an aggressive phenotype, whose molecular drivers are still under investigation (44). Simultaneously, it was shown that ZBTB46 transcription factor might act as one of these key players. It induces NGF expression upon a prolonged ADT treatment in PC patients. Mechanistically, NGF interacts with the peripheral nerve cholinergic receptor muscarinic 4 (CHRM4) to trigger PC cell differentiation by AKT and Myc-N activation. These events might finally lead to the development of neuroendocrine phenotype and ADT-resistance (18). In addition to identifying ZBTB46 as a signature for NEPC, these findings significantly contribute to the understanding of unwanted effects caused by prolonged ADT in PC patients.

Although the role of NGF and its dependent signaling in PC pathogenesis and progression is well established, genetic aberrations of NGF receptors have not been so far reported in PC (45). Thus, derangements of NGF-signaling caused by deregulation of the NGF-RTK or excessive production of NGF might be involved in PC progression. An increased expression and/or release of NGF was firstly detected in human PC specimens and PC-derived cell lines (46) and subsequently confirmed by several labs. Neurotrophic factors can be currently assayed in urine samples from PC patients (47), thus indicating a reliable, non-invasive approach for detection of novel PC biomarkers in body fluids. Extension of these 
findings to a large cohort of PC patients might expand the current strategies for patient's stratification. By contrast, RTK derangements cannot be easily detected. Nevertheless, from the findings previously discussed, it appears that some biomarkers, such as TRAF4 or ZBTB46, emerge as predictors of TrkA activation. Their overexpression correlates with aberrant TrkA activation and metastatic events or would predict, as in the case of ZBTB46, the increase in NGF levels with the subsequent signaling derangement in PC patients. These findings, together with the well-established role of neurotrophins for autonomic innervation of PC into the tumor microenvironment, indicate that NGF and their receptors are clinically actionable in PC (48 and therein refs). On the basis of preclinical findings (40,49), the RTK inhibitor, lestaurtinib (CEP-701) entered clinical trials (NCT00081601) in PC patients, with promising data from phase I studies. However, the drug failed to show a significant PSA response in patients with localized hormone-refractory PC (50). Subsequently, another small-molecule RTK inhibitor, cabozantinib was approved for the treatment of metastatic PC. Noteworthy, these and other currently used inhibitors, such as NCT02219711, block a broad range of RTK and frequently induce side-effects, mainly the drug-resistance. Thus, only indepth investigation in 3D models from PC specimens or patient's derived xenografts might allow a more tailored therapy. In this regard, the design and synthesis of small bioavailable peptides specifically perturbing the key signaling functions of NGF receptors can be envisaged. Similar approaches have been successfully applied in our lab to disrupt the upstream interactions of sex steroid receptors with signaling effectors in quite different experimental settings (15, 29, 51-55).

Lastly, it cannot be neglected the role of NGF in PC-related pain, which is the most common symptom of $\mathrm{PC}$ bone metastasis (56). Anti-NGF blocking antibodies were firstly used to reduce PC-related bone pain in mouse models. Interestingly, this pharmacologic approach showed limited adverse effects, as compared with nonselective non-steroidal drugs or opioids (57). After many years of investigations, we are now aware that neurotrophins control the autonomic innervation of tumor microenvironment and, hence, PC progression. As such, neurotrophic factor assays would predict the progression and metastatic events in PC patients. Their pharmacologic manipulation can be used to prevent $\mathrm{PC}$ progression, reduce the $\mathrm{PC}$-related bone pain and improve the quality of life in patients (48).

\section{THE ROLE OF TRANSIENT RECEPTOR POTENTIAL MELASTATIN-8 (TRPM8) IN PROSTATE CANCER}

The calcium channels mediate activation of several intracellular pathways by modulating the influx of cations (58 and therein refs). Some of them have emerged in recent years as important players in PC pathogenesis. As such, they represent 'druggable' targets in PC therapy $(59,60)$.
The transient receptor potential melastatin-8 (TRPM8) is a $\mathrm{Ca}^{2+}$-permeable cold-sensing channel, which has received increasing attention for its role in a plethora of human solid cancers, including PC (20,61-63). TRPM8 belongs to a family of eight members, classified from TRPM1 to TRPM8. They share a common structure, including a N-terminal region of almost 700 amino-acids, which endows four Melastatin Homology Regions (MHRs). MHRs are required for channel assembly and ion trafficking. The channels also exhibit a trans-membrane domain (TMD) with six helices, together with an additional intracellular helix (TRP helix). The C-terminal coiled-coil domain links the C-terminal domain (CTD) to the TRP helix (64). Figure 2 illustrates the TRPM8 molecular organization. Among the various members, TRPM8 is involved in ion's homeostasis and it is sensitive to redox-state and temperature changes. Additionally, the channel can be activated by thermal stimuli (cold), depolarization of cell membranes or chemical compounds, such as the menthol and icilin (65).

TRPM8 expression was initially discovered in sensory neurons $(66,67)$. Subsequent studies of genome wide expression profiling showed that it is abundantly expressed in prostate tissue and PC samples $(68,69)$. TRPM8 expression is regulated by androgens, while $\mathrm{ADT}$ and the androgenindependence status both reduce its expression in PC tissues (69). Again, negligible levels of TRPM8 can be detected in PC3 and DU145 cells $(22,68,70)$. These data highlight the importance of the TRPM8 channel in PC progression and hint at the usage of TRPM8 as a prognostic marker of PC progression.

Low levels of TRPM8 were detected at plasma membrane or endoplasmic reticulum of the androgen-dependent LNCaP cells. In these cells, the channel activation leads to mitogenesis by regulating $\mathrm{Ca}^{2+}$ and $\mathrm{Na}^{+}$homeostasis (70). Subsequent studies in the same cells have shown that the $\operatorname{trpm} 8$ gene is responsive to androgens, as its hormone regulation can be mediated by an androgen response element (ARE). Beyond this transcriptional regulation, a ligand-regulated degradation of TRPM8 has been detected in LNCaP cells (71). These findings underline the importance of hormone regulation in TRPM8 expression, at both transcriptional or post-translational level. Such regulation might impact the proliferation, survival and motility of PC cells. However, TRPM8 can also be regulated by PSA. In PC3 cells engineered to overexpress TRPM8, PSA acts as a channel agonist, prompting the $\mathrm{Ca}^{2+}$ intake. As such, bradykinin 2 receptor (B2R) activation occurs, with the consequent activation of protein kinase $\mathrm{C}$ pathway and inhibition of cell migration (72). Thus, once released, PSA activates TRPM8 by an autocrine loop, thereby impairing the invasive potential of PC cells. These findings point to the protective role for TRPM8 in PC. Recent findings have consistently shown that WS-12, a selective TRPM8 agonist, sensitizes the locally advanced PC to a sublethal dose of X-rays. These findings indicate that pharmacologic manipulation of TRPM8 by agonists would avoid the side effects correlated to high-dose ionizing radiation approach in PC patients (63).

We have recently reported that androgen stimulation of various PC-derived cells rapidly induces the complexation of 


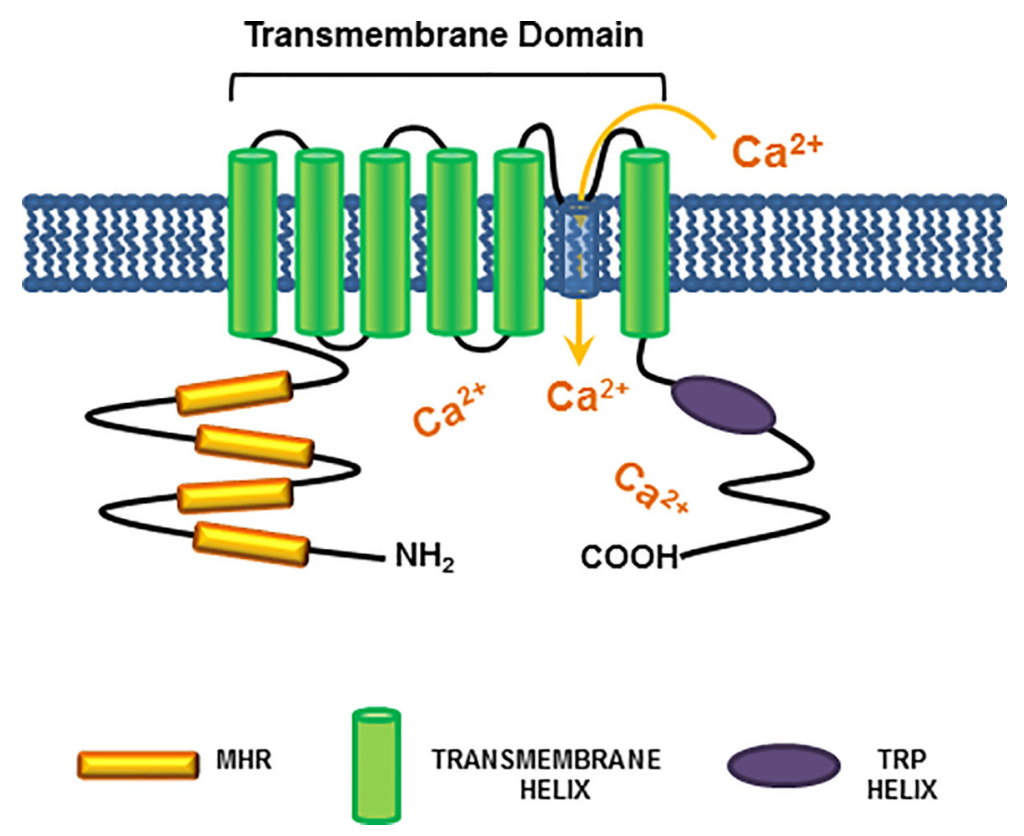

FIGURE 2 | TRPM8 structure. A schematic representation of the TRPM8 channel shows all the common structures between TRPM members. The cytosolic Nterminal region is connected to four Melastatin Homology Regions (MHRs), which are required for channel assembly and ion trafficking. The MHRs are connected to six helices within the plasma membrane, which constitute the trans-membrane domain (TMD). At last, the intracellular TRP helix is connected via a coiled-coil domain to the C-terminal domain (CTD). The indicated domains are colored as follows: MHR, orange; TMD helices: green; TRP helix: purple. As indicated in Figure, TRPM8 channel activation leads to an increase in cytosolic calcium levels.

AR with TRPM8 at extra-nuclear level. Previous findings have consistently shown that androgens trigger the AR/TRPM8 interaction within the lipid rafts microdomains of PC3 cells engineered to express AR (73). Whatever the intracellular localization of the complex, our data indicate that the androgen-induced AR/TRPM8 complex assembly controls the aggressive behaviour of PC cells through the increase in cytosolic $\left[\mathrm{Ca}^{2+}\right]$ levels. Newly synthesized TRPM8 antagonists revert these effects, impair the mitogenesis and invasion of PC cells and reduce the growth of PC cell-derived spheroids. Remarkably, the designed antagonists impair the proliferation and invasion of CRPC cells still expressing AR or the AR-V7 variant (22). As this mutant confers the anti-androgen resistance to PC patients (74), our recent study indicates that TRPM8 channel is clinically actionable in CRPC patients. In summary, we posit that TRPM8 acts as a molecular link between the androgen- and calcium-dependent signaling in PC. Therefore, the discovery of new selective TRPM8 antagonists hold promising results in PC therapy, since the lead compounds we used combine the selective modulation of AR-mediated rapid actions with the release of intracellular calcium. This combinatorial approach may be more effective than the currently used ADT. The arguments put forward here, together with the recent identification of TRPM8 mRNA as a bloodstream signature for PC aggressiveness (75), strongly encourage further studies in this direction.

The finding that TRPM8 regulates key features of PC cells call for additional comments. Other members of the same family can be regulated by sex steroids. TRPM4 and TRPM6, for instance, have been linked to non-transcriptional estrogen action in various cell types $(76,77)$. Pregnenolone sulfate, the precursor of steroid hormones, transiently activates TRPM3, thereby increasing the calcium influx and the insulin secretion from pancreatic islets (78). As such, the TRPM family members connect the steroid endocrine system with calcium and insulin pathways. Notably, NGF induces through TrkA signaling activation the up-regulation of TRPM8 in neuronal cells. This process requires the reversible activation of the Src tyrosine kinase as well as PI3-K (79), the two mainstream effectors activated by rapid actions mediated by AR in target cells. Thus, it might be argued that an intricate network made up of TrkA/ TRPM8/AR components sustains the activation of pathways triggered by NGF or androgens or calcium in PC. If that were to happen, drug escape would easily occur. As such, ADT or TrkA inhibitors or even TRPM8 antagonists might fail in monotherapy.

\section{THE ROLE OF CANCER ASSOCIATED FIBROBLASTS IN PROSTATE CANCER PATHOGENESIS AND PROGRESSION}

CAFs represent the most important component of the tumor microenvironment (TME), which surrounds the neoplastic tissue. Together with the extracellular matrix (ECM), blood vessels and immune cells, CAFs have emerged as key regulators of cancer cell proliferation and metastasis. They 
respond to the tumor-released growth factors or cytokines and secrete cytokines, chemokines and growth factors of their own. Again, by depositing or degrading ECM proteins, CAFs also influence the TME architecture, thus creating a favorable or a disadvantageous environment for the onset and progression of several tumors. The study of CAFs origin and analysis of their actions have emerged during the last years as a main road for a better understanding of cancer pathogenesis and progression, as well as the design of novel strategies for diagnostic and therapeutic guidance in patients (reviewed in 80,81).

In normal tissues, fibroblasts contribute to the production of ECM and are major players in restoring the tissue integrity upon injury or chronic damage. Under such conditions, they acquire an activated phenotype, characterized by the expression of a subset of mesenchymal markers, including $\alpha$-smooth muscle actin ( $\alpha$-SMA), fibroblast activating protein (FAP) and vimentin $(82,83)$. At this stage, they become myofibroblasts, contribute to the tissue recovery by releasing cytokines, chemokines and growth factors, such as the TGF- $\beta$ and the vascular endothelial growth factor A (VEGF-A), and also recruit immune cells. CAFs derived from neoplastic tissues lack the markers for epithelial/ endothelial lineages, show a decrease in CD36 expression and exhibit few genetic mutations, in the presence of an elongated cell morphology. They can be detected before the onset of a proper neoplasia, as a consequence of a tissue injury or damage. However, CAFs may also arise from a population of fibroblasts surrounding primitive lesions, with the initial aim to suppress the tumor growth. Additional events might switch this function, addressing them towards a tumor-supporting activity. Other studies have also indicated a possible, though less likely, origin of CAFs from mesenchymal stem cells, pericytes or adipocytes in different tumor types. Finally, CAF activation typically depends on soluble molecules, including interleukins and growth factors, which activate several intracellular signaling pathways, such as the TGF- $\beta$, Notch and NF- $\mathrm{BB}$ ones. Direct contact between cancer cells and fibroblasts could be also responsible for their activation, even if only in some cancer types. Simultaneously, other TME components could induce fibroblasts activation, regardless of cancer cells presence $(81,84)$.

Pioneering studies have pointed to the fundamental role of CAFs in supporting the onset of PC (85). Currently available data indicate that prostate CAFs establish a reciprocal, paracrine interaction with PC cells, resulting in EMT of the latter. Interleukin-6 (IL-6) produced by PC3 cells activates fibroblasts derived from patients with benign prostatic hyperplasia (BPH). Interestingly, different markers of fibroblast activation can be detected in these conditions, as compared with those expressed upon TGF- $\beta$-mediated activation (86). At the same time, CAFs activated by IL- 6 promote EMT of PC3 cells and increase invasiveness in a similar fashion to that achieved by TGF- $\beta$ activated fibroblasts. Interestingly, only transformed prostate cells trigger CAFs activation, and such skill strictly depends on cell aggressiveness. Unlike PC3 or DU145, androgen-sensitive LNCaP cells seem, indeed, unable to activate CAFs. The mechanism by which PC cells acquire invasive phenotype, together with the EMT, depends on secretion of matrix metalloproteinases 2 and 9 (MMP2, MMP9) by CAFs. This response can be only detected on IL-6 stimulation and is reversed by MMPs inhibitors, thus indicating a role for MMPs in the observed findings. Additionally, CAFs are required for PC onset and metastatic spreading in mouse xenograft models, since their absence impairs the PC3 cell ability to generate subcutaneous tumors. Again, CAF-induced EMT of PC cells contributes to the formation of PC stem cells, thus enhancing cancer stemness (87). CAFs also play an energy-supporting role in PC, since prolonged exposure of PC3 and DU145 cells to CAF-conditioned media increases PC cell mitochondrial activity through sirtuin 1 /peroxisome proliferator-activated receptor gamma coactivator $1-\alpha$ (SIRT1/PGC-1 $\alpha$ ) axis. SIRT1 or PGC$1 \alpha$ silencing impairs the CAF-induced EMT in PC3 cells. Moreover, metabolic deregulation of PC cells by CAFs ultimately increases PC cells invasive potential by stabilization of hypoxia-induced factor $1 \alpha$ (HIF- $1 \alpha$ ), a reported feature of enhanced PC cell malignancy. Additionally, CAFs are also able to transfer horizontally and unidirectionally their dispensable, functional mitochondria to PC cells, thus contributing to their increased metabolic capacity, both directly or through activation of the SIRT1/PGC-1 $\alpha$ pathway. By this mechanism, CAFs establish a symbiotic interaction with PC cells, which sustains their proliferative and metastatic behavior through metabolic regulation, production of high-energy metabolites and mitochondria supply (88). Recent findings have also indicated a role for CAFs in the development of castration-resistance. Accordingly, $\mathrm{PC}$ resistance to the $2^{\text {nd }}$ generation antiandrogen, enzalutamide, would depend on CAFs, as shown in a PC mouse model progressing from castration-sensitive to castrationresistant state. The process seems mediated by the activation of the specific NRG1 receptor, HER3, by CAFs-secreted neuregulin-1 (NRG-1). Altogether, the proposed findings identify the NRG1/HER3 axis as a main candidate in the antiandrogen resistance. In support of this concept, high levels of NRG1, together with an enhanced NRG1 mRNA expression, can be detected in CAFs from ADT-treated PC patients (17). These results suggest that targeting of the NRG1/HER3 axis may be beneficial in CRPC patients. Figure 3 schematically depicts the interplay between CAFs and PC cells.

However, the findings so far presented raise an important question. It concerns the expression of AR in CAFs. Previous findings from prostate CAFs have shown that the receptor is expressed at lower levels, as compared with LNCaP cells. Its somatic knockdown reduced the proliferative and migratory potential of PC3 cells, suggesting a role for stromal AR in sustaining the growth and invasion of PC cells (24). By contrast, subsequent studies reported that inhibition of $A R$ in murine CAFs increases the expression of stemness markers in co-cultured PC cells. This effect was attributed to the release of interferon- $\gamma($ IFN- $\gamma$ ) and macrophage colony stimulating factor (M-CSF) by AR-depleted murine CAFs (25). Consistent with a protective role for stromal $\mathrm{AR}$, it has been reported that expression of the receptor reduces the release of CCL2 and CXCL8 by CAFs, thereby inhibiting PC cell motility (89). These findings further corroborate the idea that PC 


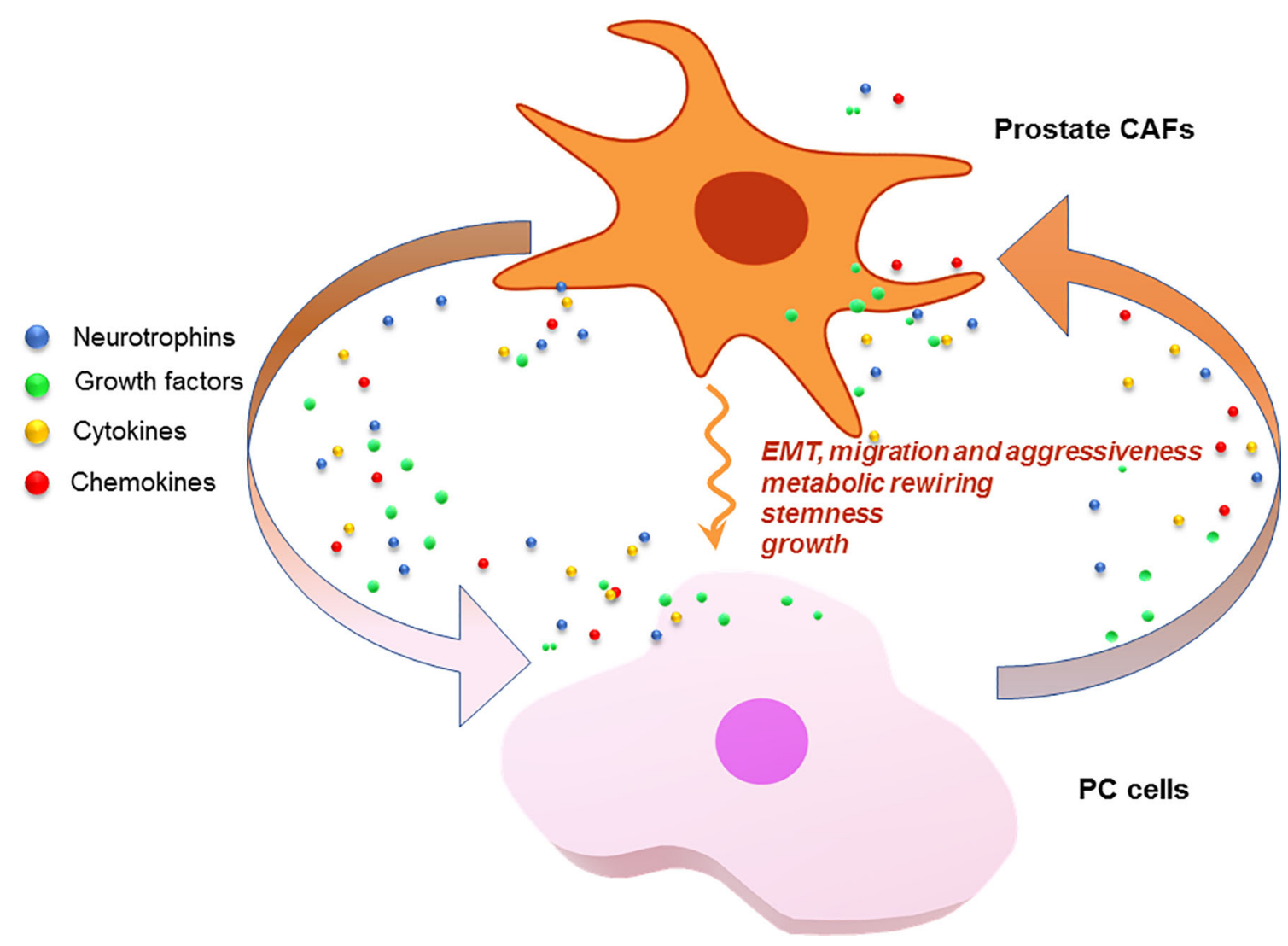

FIGURE 3 | CAFs and PC cells as signal exchangers. CAFs or PC cells release neurotrophins, growth factors, cytokines and chemokines. These signals are exchanged by the cells to foster a plethora of responses that lead to PC progression and drug-resistance.

progression might be paradoxically fostered by ADT or AR blocking therapies. Additional findings have supported an inverse correlation between stromal AR and disease's malignancy. Immunohistochemistry (IHC) analysis has shown, indeed, that expression of $\mathrm{AR}$ is more abundant in nonmalignant stroma, as compared with PC-related stroma, further suggesting a protective and antioncogenic role for stromal AR (90-92). In contrast with these findings, our recent study in short-term primary culture of CAFs derived from PC specimens has revealed low, but appreciable AR levels in almost all the CAFs analyzed, even in about 30\% of CAFs from PC patients at high Gleason's score (29). The conflicting findings so far reported might be explained by different considerations. Firstly, the IHC approach for detection of sex steroid receptors often exhibits pitfalls because of the type of cell permeabilization or the primary antibody used (93). Again, AR might be lost in stromal cells as a consequence of CAF selection and/or cell culture manipulation. Additionally, the receptor might undergo degradation as a consequence of ubiquitinproteasome pathway activation (94) or epigenetic changes (95). Whatever the case, many studies support an oncogenic role for stromal AR, which might induce prostatic intraepithelial neoplasia (PIN; 96) or even metastatic events (97). We have consistently reported that stromal AR directs CAFs towards PC epithelial cells. This process requires AR complexation with filamin A (FlnA) and is strongly stimulated by androgens in 2 and $3 \mathrm{D}$ cell culture models. As such, a significant increase in PCderived organoid's size can be detected (29). A similar process might occur in vivo when the local androgen levels increase, as it frequently occurs in PC (98). Nevertheless, the role of other factors, including NGF, cannot be neglected. In such a way, stromal AR might change the TME composition and allow metastatic events. In support of a role for stromal AR in cancer aggressiveness, it has been shown that AR targeting in CAFs reduces skin cancer aggressiveness traits (26) or even inhibit the development of chemo-resistant skin cancers (27). Thus, ARdirected therapies in CAFs might help the therapeutic approach of different cancer types. We designed and successfully used a small modified peptide that perturbs the androgen-induced AR/ FlnA complex assembly (53). By this way, the peptide inhibits the androgen-induced invasion of CAFs in 2D models and reduces the overall tumor size in androgen-treated 3D co-culture (29). Our translational findings identify the AR/FlnA complex as a new 'druggable' biomarker in prostate CAFs. The strategy we propose might be profitably used for a more efficient PC treatment. 


\section{CONCLUDING REMARKS}

PC still represents the second leading cause of cancer death in men in Western society. In most cases ( 70\%), PC has a slow and symptom-free growth, whereas it is more aggressive in the remaining patients. Current therapies for advanced PC remain unsatisfactory and new inhibitors of androgen synthesis and AR activation have been designed to improve patient survival. Despite these therapies, PC often progress towards a metastatic and/or CRPC phenotype. Preclinical and clinical studies currently aim at identifying the molecular basis for PC spreading and drug-resistance. Nevertheless, few biomarkers predictive of metastatic phenotype have as yet been identified and few efficient therapeutic options are available for advanced PC. Recent years, have seen important advances in large scale omics approaches to identify novel biomarkers of PC malignancy and ameliorate patients' stratification as well as clinical management of the disease. The combination of next generation sequencing (NGS) approaches with proteomic profiling has revealed important differences between malignant and benign specimens, together with the identification of clinically actionable biomarkers. Additionally, tools for transcriptional expression classification have allowed the PCs characterization based on their pathological features, thereby addressing the patients towards a proper care (99). Several epigenetic modifications have been also identified and linked to altered gene expression, resulting in increased PC risk. Transcriptome-wide association studies confirmed the correlation with the expression of several predicted genes (100). Similar tools have been also applied to prostate CAFs to show that increased expression of ECM remodelling proteins is linked to cancer-supporting properties (101) or that specific epigenetic alterations are correlated to PC malignancy (102). These approaches might further provide important information on PC molecular landscape.

In this review, we aimed to address novel aspects of PC biology, which impinge on the interconnections between AR and other key intracellular signalling regulated by NGF or calcium channels. Communications between these partners seem relevant, since their breakdown might underly PC pathogenesis and progression. Current PC therapies prevalently target proliferative functions of $\mathrm{AR}$ and may only be effective within a short time frame. Primary PC show, instead, a marked heterogeneity and tumor cells may rapidly change as a consequence of pressures exerted by CAFs. Thus, further analysis of tumor microenvironment, identification of its molecular signatures, including the AR expression and its main partners, together with in depth study of signals delivered by PCor CAF-derived exosomes (103), would provide additional information for patient's stratification, avoiding expensive therapies with considerable side effects. In this context, the synthesis of new biologically active molecules, such as the new calcium-channel antagonists or the small bioavailable peptides, specifically perturbing the extranuclear AR functions in TME or PC cells, should improve the pharmacologic response in patients and ameliorate their quality of life.

\section{AUTHOR CONTRIBUTIONS}

FL and GC conceptualized the paper and performed the initial and final editing. FL, MD, and AnM conceptualized and arranged the Figure's images. All the authors contributed to paper writing and revision. All authors contributed to the article and approved the submitted version.

\section{FUNDING}

This work was supported by Italian Ministry of University and Scientific Research (P.R.I.N. 2017EKMFTN_002 to GC); VALERE Program (Vanvitelli per la Ricerca Program; GoMAGIC to A.M., AdipCARE to GC and DESIRE to PG). Marzia Di Donato is supported by iCURE Project (B21C17000030007- Regione Campania).

\section{REFERENCES}

1. Sung H, Ferlay J, Siegel RL, Laversanne M, Soerjomataram I, Jemal A, et al. Global Cancer Statistics 2020: GLOBOCAN Estimates of Incidence and Mortality Worldwide for 36 Cancers in 185 Countries. CA: Cancer J Clin (2021) 71(3):209-49. doi: 10.3322/caac.21660

2. Pernar CH, Ebot EM, Wilson KM, Mucci LA. The Epidemiology of Prostate Cancer. Cold Spring Harbor Perspect Med (2018) 8(12):a030361. doi: 10.1101/cshperspect.a030361

3. Cuccurullo V, Di Stasio GD, Evangelista L, Castoria G, Mansi L. Biochemical and Pathophysiological Premises to Positron Emission Tomography With Choline Radiotracers. JCell Physiol (2017) 232(2):270-5. doi: 10.1002/jcp.25478

4. Miralbell R. Stereotactic Beam Radiotherapy for Prostate Cancer: Is Less, More? Lancet Oncol (2019) 20(11):1471-2. doi: 10.1016/S1470-2045(19)30652-7

5. Siegel RL, Miller KD, Fuchs H, Jemal A. Cancer Statistic, 2021. CA: A Cancer J Clin (2021) 71:7- 33. doi: 10.3322/caac.21654

6. Attard G, Parker C, Eeles RA, Schröder F, Tomlins SA, Tannock I, et al. Prostate Cancer. Lancet (London England) (2016) 387(10013):70-82. doi: 10.1016/S0140-6736(14)61947-4

7. Nuhn P, De Bono JS, Fizazi K, Freedland SJ, Grilli M, Kantoff, et al. Update on Systemic Prostate Cancer Therapies: Management of Metastatic Castration-Resistant Prostate Cancer in the Era of Precision Oncology. Eur Urol (2019) 75(1):88-99. doi: 10.1016/j.eururo.2018.03.028

8. Davies AH, Beltran H, Zoubeidi A. Cellular Plasticity and the Neuroendocrine Phenotype in Prostate Cancer. Nat Rev Urol (2018) 15 (5):271-86. doi: 10.1038/nrurol.2018.22

9. Shen MM, Abate-Shen C. Molecular Genetics of Prostate Cancer: New Prospects for Old Challenges. Genes Dev (2010) 24(18):1967-2000. doi: 10.1101/gad.1965810

10. Logothetis CJ, Gallick GE, Maity SN, Kim J, Aparicio A, Efstathiou E, et al. Molecular Classification of Prostate Cancer Progression: Foundation for Marker-Driven Treatment of Prostate Cancer. Cancer Discov (2013) 3 (8):849-61. doi: 10.1158/2159-8290.CD-12-0460

11. Howard N, Clementino M, Kim D, Wang L, Verma A, Shi X, et al. New Developments in Mechanisms of Prostate Cancer Progression. Semin Cancer Biol (2019) 57:111-6. doi: 10.1016/j.semcancer.2018.09.003

12. Luo J, Attard G, Balk SP, Bevan C, Burnstein K, Cato L, et al. Role of Androgen Receptor Variants in Prostate Cancer: Report From the 2017 
Mission Androgen Receptor Variants Meeting. Eur Urol (2018) 73(5):71523. doi: 10.1016/j.eururo.2017.11.038

13. Huang EJ, Reichardt LF. Trk Receptors: Roles in Neuronal Signal Transduction. Annu Rev Biochem (2003) 72:609-42. doi: 10.1146/ annurev.biochem.72.121801.161629

14. Singh R, Karri D, Shen H, Shao J, Dasgupta S, Huang S, et al. TRAF4Mediated Ubiquitination of NGF Receptor Trka Regulates Prostate Cancer Metastasis. J Clin Invest (2018) 128(7):3129-43. doi: 10.1172/JCI96060

15. Di Donato M, Cernera G, Auricchio F, Migliaccio A, Castoria G. Cross-Talk Between Androgen Receptor and Nerve Growth Factor Receptor in Prostate Cancer Cells: Implications for a New Therapeutic Approach. Cell Death Discovery (2018) 4:5. doi: 10.1038/s41420-017-0024-3

16. Di Donato M, Cernera G, Migliaccio A, Castoria G. Nerve Growth Factor Induces Proliferation and Aggressiveness in Prostate Cancer Cells. Cancers (2019) 11(6):784. doi: 10.3390/cancers11060784

17. Zhang Z, Karthaus WR, Lee YS, Gao VR, Wu C, Russo JW, et al. Tumor Microenvironment-Derived NRG1 Promotes Antiandrogen Resistance in Prostate Cancer. Cancer Cell (2020) 38(2):279-296.e9. doi: 10.1016/ j.ccell.2020.06.005

18. Chen WY, Wen YC, Lin SR, Yeh HL, Jiang KC, Chen WH, et al. Nerve Growth Factor Interacts With CHRM4 and Promotes Neuroendocrine Differentiation of Prostate Cancer and Castration Resistance. Commun Biol (2021) 4(1):22. doi: 10.1038/s42003-020-01549-1

19. Zhang L, Barritt GJ. TRPM8 in Prostate Cancer Cells: A Potential Diagnostic and Prognostic Marker With a Secretory Function? Endoc Relat Cancer (2006) 13(1):27-38. doi: 10.1677/erc.1.01093

20. Grolez GP, Gkika D. TRPM8 Puts the Chill on Prostate Cancer. Pharmaceuticals (Basel Switzerland) (2016) 9(3):44. doi: 10.3390/ph9030044

21. Alaimo A, De Felice D, Genovesi S, Lorenzoni M, Lunardi A. Tune the Channel: TRPM8 Targeting in Prostate Cancer. Oncoscience (2021) 8:97100. doi: $10.18632 /$ oncoscience. 543

22. Di Donato M, Ostacolo C, Giovannelli P, Di Sarno V, Monterrey I, Campiglia P, et al. Therapeutic Potential of TRPM8 Antagonists in Prostate Cancer. Sci Rep (2021) 11(1):23232. doi: 10.1038/s41598-02102675-4

23. Cioni B, Zwart W, Bergman AM. Androgen Receptor Moonlighting in the Prostate Cancer Microenvironment. Endoc Relat Cancer (2018) 25(6):R33149. doi: 10.1530/ERC-18-0042

24. Yu S, Xia S, Yang D, Wang K, Yeh S, Gao Z, et al. Androgen Receptor in Human Prostate Cancer-Associated Fibroblasts Promotes Prostate Cancer Epithelial Cell Growth and Invasion. Med Oncol (Northwood London England) (2013) 30(3):674. doi: 10.1007/s12032-013-0674-9

25. Liao CP, Chen LY, Luethy A, Kim Y, Kani K, MacLeod AR, et al. Androgen Receptor in Cancer-Associated Fibroblasts Influences Stemness in Cancer Cells. Endoc Relat Cancer (2017) 24(4):157-70. doi: 10.1530/ERC-16-0138

26. Clocchiatti A, Ghosh S, Procopio MG, Mazzeo L, Bordignon P, Ostano P, et al. Androgen Receptor Functions as Transcriptional Repressor of CancerAssociated Fibroblast Activation. J Clin Invest (2018) 128(12):5531-48. doi: $10.1172 /$ JCI99159

27. Chan J, Sng MK, Teo ZQ, Chong HC, Twang JS, Tan NS. Targeting Nuclear Receptors in Cancer-Associated Fibroblasts as Concurrent Therapy to Inhibit Development of Chemoresistant Tumors. Oncogene (2018) 37 (2):160-73. doi: 10.1038/onc.2017.319

28. Mishra R, Haldar S, Suchanti S, Bhowmick NA. Epigenetic Changes in Fibroblasts Drive Cancer Metabolism and Differentiation. Endoc Relat Cancer (2019) 26(12):R673-88. doi: 10.1530/ERC-19-0347

29. Di Donato M, Zamagni A, Galasso G, Di Zazzo E, Giovannelli P, Barone MV, et al. The Androgen Receptor/Filamin a Complex as a Target in Prostate Cancer Microenvironment. Cell Death Dis (2021) 12(1):127. doi: 10.1038/s41419-021-03402-7

30. Dollé L, El Yazidi-Belkoura I, Adriaenssens E, Nurcombe V, Hondermarck H. Nerve Growth Factor Overexpression and Autocrine Loop in Breast Cancer Cells. Oncogene (2003) 22(36):5592-601. doi: 10.1038/sj.onc.1206805

31. Pundavela J, Roselli S, Faulkner S, Attia J, Scott RJ, Thorne RF, et al. Nerve Fibers Infiltrate the Tumor Microenvironment and are Associated With Nerve Growth Factor Production and Lymph Node Invasion in Breast Cancer. Mol Oncol (2015) 9(8):1626-35. doi: 10.1016/j.molonc. 2015.05.001
32. Chakravarthy R, Mnich K, Gorman AM. Nerve Growth Factor (NGF)Mediated Regulation of P75(NTR) Expression Contributes to Chemotherapeutic Resistance in Triple Negative Breast Cancer Cells. Biochem Biophys Res Commun (2016) 478(4):1541-7. doi: 10.1016/ j.bbrc.2016.08.149

33. Di Donato M, Galasso G, Giovannelli P, Sinisi AA, Migliaccio A, Castoria G. Targeting the Nerve Growth Factor Signaling Impairs the Proliferative and Migratory Phenotype of Triple-Negative Breast Cancer Cells. Front Cell Dev Biol (2021) 9:676568. doi: 10.3389/fcell.2021.676568

34. Cheng B, Mattson MP. NGF and Bfgf Protect Rat Hippocampal and Human Cortical Neurons Against Hypoglycemic Damage by Stabilizing Calcium Homeostasis. Neuron (1991) 7(6):1031-41. doi: 10.1016/0896-6273(91) 90347-3

35. Huang J, Ye Z, Hu X, Lu L, Luo Z. Electrical Stimulation Induces CalciumDependent Release of NGF From Cultured Schwann Cells. Glia (2010) 58 (5):622-31. doi: 10.1002/glia.20951

36. Jobling P, Pundavela J, Oliveira SM, Roselli S, Walker MM, Hondermarck H. Nerve-Cancer Cell Cross-Talk: A Novel Promoter of Tumor Progression. Cancer Res (2015) 75(9):1777-81. doi: 10.1158/0008-5472.CAN-14-3180

37. Meldolesi J. Neurotrophin Trk Receptors: New Targets for Cancer Therapy. Rev Physiol Biochem Pharmacol (2018) 174:67-79. doi: 10.1007/112_2017_6

38. Geldof AA, Van Haarst EP, Newling DW. Neurotrophic Factors in Prostate and Prostatic Cancer. Prostate Cancer Prostatic Dis (1998) 1(5):236-41. doi: 10.1038/sj.pcan.4500247

39. Sortino MA, Condorelli F, Vancheri C, Chiarenza A, Bernardini R, Consoli U, et al. Mitogenic Effect of Nerve Growth Factor (NGF) in Lncap Prostate Adenocarcinoma Cells: Role of the High- and Low-Affinity NGF Receptors. Mol Endocrinol (Baltimore Md) (2000) 14(1):124-36. doi: 10.1210/ mend.14.1.0402

40. Festuccia C, Muzi P, Gravina GL, Millimaggi D, Speca S, Dolo V, et al. Tyrosine Kinase Inhibitor CEP-701 Blocks the NTRK1/NGF Receptor and Limits the Invasive Capability of Prostate Cancer Cells In Vitro. Int J Oncol (2007) 30(1):193-200. doi: 10.3892/ijo.30.1.193

41. Arrighi N, Bodei S, Zani D, Simeone C, Cunico SC, Missale C, et al. Nerve Growth Factor Signaling in Prostate Health and Disease. Growth Factors (Chur Switzerland) (2010) 28(3):191-201. doi: 10.3109/08977190903578678

42. Bradshaw RA, Pundavela J, Biarc J, Chalkley RJ, Burlingame AL, Hondermarck H. NGF and Prongf: Regulation of Neuronal and Neoplastic Responses Through Receptor Signaling. Adv Biol Regul (2015) 58:16-27. doi: 10.1016/j.jbior.2014.11.003

43. Dardenne E, Beltran H, Benelli M, Gayvert K, Berger A, Puca L, et al. N-Myc Induces an EZH2-Mediated Transcriptional Program Driving Neuroendocrine Prostate Cancer. Cancer Cell (2016) 30(4):563-77. doi: $10.1016 /$ j.ccell.2016.09.005

44. Wang Y, Wang Y, Ci X, Choi S, Crea F, Lin D, et al. Molecular Events in Neuroendocrine Prostate Cancer Development. Nat Rev Urol (2021) 18 (10):581-96. doi: 10.1038/s41585-021-00490-0

45. Rosen EY, Goldman DA, Hechtman JF, Benayed R, Schram AM, Cocco E, et al. TRK Fusions are Enriched in Cancers With Uncommon Histologies and the Absence of Canonical Driver Mutations. Clin Cancer Res an OffJ Am Assoc Cancer Res (2020) 26(7):1624-32. doi: 10.1158/1078-0432.CCR-193165

46. Dalal R, Djakiew D. Molecular Characterization of Neurotrophin Expression and the Corresponding Tropomyosin Receptor Kinases (Trks) in Epithelial and Stromal Cells of the Human Prostate. Mol Cell Endocrinol (1997) 134 (1):15-22. doi: 10.1016/s0303-7207(97)00165-2

47. March B, Lockhart KR, Faulkner S, Smolny M, Rush R, Hondermarck H. ELISA-Based Quantification of Neurotrophic Growth Factors in Urine From Prostate Cancer Patients. FASEB bioAdvances (2021) 3(11):888-96. doi: 10.1096/fba.2021-00085

48. March B, Faulkner S, Jobling P, Steigler A, Blatt A, Denham J, et al. Tumour Innervation and Neurosignalling in Prostate Cancer. Nat Rev Urol (2020) 17 (2):119-30. doi: 10.1038/s41585-019-0274-3

49. Weeraratna AT, Dalrymple SL, Lamb JC, Denmeade SR, Miknyoczki S, Dionne CA, et al. Pan-Trk Inhibition Decreases Metastasis and Enhances Host Survival in Experimental Models as a Result of its Selective Induction of Apoptosis of Prostate Cancer Cells. Clin Cancer Res an Off J Am Assoc Cancer Res (2001) 7(8):2237-45. 
50. Collins C, Carducci MA, Eisenberger MA, Isaacs JT, Partin AW, Pili R, et al. Preclinical and Clinical Studies With the Multi-Kinase Inhibitor CEP-701 as Treatment for Prostate Cancer Demonstrate the Inadequacy of PSA Response as a Primary Endpoint. Cancer Biol Ther (2007) 6(9):1360-7. doi: $10.4161 /$ cbt.6.9.4541

51. Migliaccio A, Castoria G, Auricchio F. Src-Dependent Signalling Pathway Regulation by Sex-Steroid Hormones: Therapeutic Implications. Int J Biochem Cell Biol (2007) 39(7-8):1343-8. doi: 10.1016/j.biocel.2006.12.009

52. Varricchio L, Migliaccio A, Castoria G, Yamaguchi H, de Falco A, Di Domenico M, et al. Inhibition of Estradiol Receptor/Src Association and Cell Growth by an Estradiol Receptor Alpha Tyrosine-Phosphorylated Peptide. Mol Cancer Res MCR (2007) 5(11):1213-21. doi: 10.1158/1541-7786.MCR07-0150

53. Castoria G, Giovannelli P, Di Donato M, Ciociola A, Hayashi R, Bernal F, et al. Role of non-Genomic Androgen Signalling in Suppressing Proliferation of Fibroblasts and Fibrosarcoma Cells. Cell Death Dis (2014) 5(12):e1548. doi: $10.1038 /$ cddis. 2014.497

54. Di Donato M, Bilancio A, D’Amato L, Claudiani P, Oliviero MA, Barone MV, et al. Cross-Talk Between Androgen Receptor/Filamin a and Trka Regulates Neurite Outgrowth in PC12 Cells. Mol Biol Cell (2015) 26 (15):2858-72. doi: 10.1091/mbc.E14-09-1352

55. Di Donato M, Giovannelli P, Barone MV, Auricchio F, Castoria G, Migliaccio A. A Small Peptide Targeting the Ligand-Induced Androgen Receptor/Filamin a Interaction Inhibits the Invasive Phenotype of Prostate Cancer Cells. Cells (2021) 11(1):14. doi: 10.3390/cells11010014

56. Coleman RE. Skeletal Complications of Malignancy. Cancer (1997) 80(8 Suppl):1588-94. doi: 10.1002/(sici)1097-0142(19971015)80:8+<1588::aidcncr9>3.3.co; $2-\mathrm{z}$

57. Halvorson KG, Kubota K, Sevcik MA, Lindsay TH, Sotillo JE, Ghilardi JR, et al. A Blocking Antibody to Nerve Growth Factor Attenuates Skeletal Pain Induced by Prostate Tumor Cells Growing in Bone. Cancer Res (2005) 65 (20):9426-35. doi: 10.1158/0008-5472.CAN-05-0826

58. Gautier M, Trebak M, Fleig A, Vandier C, Ouadid-Ahidouch H. $\mathrm{Ca}^{2+}$ Channels in Cancer. Cell Calcium (2019) 84:102083. doi: 10.1016/ j.ceca.2019.102083

59. Yu S, Xu Z, Zou C, Wu D, Wang Y, Yao X, et al. Ion Channel TRPM8 Promotes Hypoxic Growth of Prostate Cancer Cells via an O2 -Independent and RACK1-Mediated Mechanism of HIF-1 $\alpha$ Stabilization. J Pathol (2014) 234(4):514-25. doi: 10.1002/path.4413

60. Maly IV, Hofmann WA. Calcium and Nuclear Signaling in Prostate Cancer. Int J Mol Sci (2018) 19(4):1237. doi: 10.3390/ijms19041237

61. Wondergem R, Ecay TW, Mahieu F, Owsianik G, Nilius B. HGF/SF and Menthol Increase Human Glioblastoma Cell Calcium and Migration. Biochem Biophys Res Commun (2008) 372(1):210-5. doi: 10.1016/ j.bbrc.2008.05.032

62. Liu Z, Wu H, Wei Z, Wang X, Shen P, Wang S, et al. TRPM8: A Potential Target for Cancer Treatment. J Cancer Res Clin Oncol (2016) 142(9):187181. doi: 10.1007/s00432-015-2112-1

63. Alaimo A, Lorenzoni M, Ambrosino P, Bertossi A, Bisio A, Macchia A, et al. Calcium Cytotoxicity Sensitizes Prostate Cancer Cells to Standard-of-Care Treatments for Locally Advanced Tumors. Cell Death Dis (2020) 11 (12):1039. doi: 10.1038/s41419-020-03256-5

64. Huang Y, Fliegert R, Guse AH, Lü W, Du J. A Structural Overview of the Ion Channels of the TRPM Family. Cell Calcium (2020) 85:102111. doi: 10.1016/ j.ceca.2019.102111

65. Yin Y, Wu M, Zubcevic L, Borschel WF, Lander GC, Lee SY. Structure of the Cold- and Menthol-Sensing Ion Channel TRPM8. Sci (New York NY) (2018) 359(6372):237-41. doi: 10.1126/science.aan4325

66. McKemy DD, Neuhausser WM, Julius D. Identification of a Cold Receptor Reveals a General Role for TRP Channels in Thermosensation. Nature (2002) 416(6876):52-8. doi: 10.1038/nature719

67. Peier AM, Moqrich A, Hergarden AC, Reeve AJ, Andersson DA, Story GM, et al. A TRP Channel That Senses Cold Stimuli and Menthol. Cell (2002) 108 (5):705-15. doi: 10.1016/s0092-8674(02)00652-9

68. Tsavaler L, Shapero MH, Morkowski S, Laus R. Trp-P8, a Novel ProstateSpecific Gene, is Up-Regulated in Prostate Cancer and Other Malignancies and Shares High Homology With Transient Receptor Potential Calcium Channel Proteins. Cancer Res (2001) 61(9):3760-9.
69. Henshall SM, Afar DE, Hiller J, Horvath LG, Quinn DI, Rasiah KK, et al. Survival Analysis of Genome-Wide Gene Expression Profiles of Prostate Cancers Identifies New Prognostic Targets of Disease Relapse. Cancer Res (2003) 63(14):4196-203.

70. Zhang L, Barritt GJ. Evidence That TRPM8 is an Androgen-Dependent Ca2+ Channel Required for the Survival of Prostate Cancer Cells. Cancer Res (2004) 64(22):8365-73. doi: 10.1158/0008-5472.CAN-04-2146

71. Asuthkar S, Velpula KK, Elustondo PA, Demirkhanyan L, Zakharian E. TRPM8 Channel as a Novel Molecular Target in Androgen-Regulated Prostate Cancer Cells. Oncotarget (2015) 6(19):17221-36. doi: 10.18632/ oncotarget. 3948

72. Gkika D, Flourakis M, Lemonnier L, Prevarskaya N. PSA Reduces Prostate Cancer Cell Motility by Stimulating TRPM8 Activity and Plasma Membrane Expression. Oncogene (2010) 29(32):4611-6. doi: 10.1038/onc.2010.210

73. Grolez GP, Gordiendko DV, Clarisse M, Hammadi M, Desruelles E, Fromont G, et al. TRPM8-Androgen Receptor Association Within Lipid Rafts Promotes Prostate Cancer Cell Migration. Cell Death Dis (2019) 10 (9):652. doi: 10.1038/s41419-019-1891-8

74. Antonarakis ES, Lu C, Wang H, Luber B, Nakazawa M, Roeser JC, et al. ARV7 and Resistance to Enzalutamide and Abiraterone in Prostate Cancer. New Engl J Med (2014) 371(11):1028-38. doi: 10.1056/NEJMoa1315815

75. Souza MF, Kuasne H, Barros-Filho MC, Cilião HL, Marchi FA, Fuganti PE, et al. Circulating Mrna Signature as a Marker for High-Risk Prostate Cancer. Carcinogenesis (2020) 41(2):139-45. doi: 10.1093/carcin/bgz129

76. Cao G, van der Wijst J, van der Kemp A, van Zeeland F, Bindels RJ, Hoenderop JG. Regulation of the Epithelial Mg2+ Channel TRPM6 by Estrogen and the Associated Repressor Protein of Estrogen Receptor Activity (REA). J Biol Chem (2009) 284(22):14788-95. doi: 10.1074/jbc.M808752200

77. Wong KK, Hussain FA. TRPM4 Is Overexpressed in Breast Cancer Associated With Estrogen Response and Epithelial-Mesenchymal Transition Gene Sets. PloS One (2020) 15(6):e0233884. doi: 10.1371/ journal.pone.0233884

78. Wagner TF, Loch S, Lambert S, Straub I, Mannebach S, Mathar I, et al. Transient Receptor Potential M3 Channels are Ionotropic Steroid Receptors in Pancreatic Beta Cells. Nat Cell Biol (2008) 10(12):1421-30. doi: 10.1038/ ncb1801

79. Kayama Y, Shibata M, Takizawa T, Ibata K, Nakahara J, Shimizu T, et al. Signaling Pathways Relevant to Nerve Growth Factor-Induced Upregulation of Transient Receptor Potential M8 Expression. Neuroscience (2017) 367:178-88. doi: 10.1016/j.neuroscience.2017.10.037

80. Kalluri R. The Biology and Function of Fibroblasts in Cancer. Nat Rev Cancer (2016) 16(9):582-98. doi: 10.1038/nrc.2016.73

81. Sahai E, Astsaturov I, Cukierman E, DeNardo DG, Egeblad M, Evans RM, et al. A Framework for Advancing Our Understanding of Cancer-Associated Fibroblasts. Nat Rev Cancer (2020) 20(3):174-86. doi: 10.1038/s41568-0190238-1

82. Tomasek JJ, Gabbiani G, Hinz B, Chaponnier C, Brown RA. Myofibroblasts and Mechano-Regulation of Connective Tissue Remodelling. Nat Rev Mol Cell Biol (2002) 3(5):349-63. doi: 10.1038/nrm809

83. Roberts EW, Deonarine A, Jones JO, Denton AE, Feig C, Lyons SK, et al. Depletion of Stromal Cells Expressing Fibroblast Activation Protein- $\alpha$ From Skeletal Muscle and Bone Marrow Results in Cachexia and Anemia. J Exp Med (2013) 210(6):1137-51. doi: 10.1084/jem.20122344

84. Gascard P, Tlsty TD. Carcinoma-Associated Fibroblasts: Orchestrating the Composition of Malignancy. Genes Dev (2016) 30(9):1002-19. doi: 10.1101/ gad.279737.116

85. Olumi AF, Grossfeld GD, Hayward SW, Carroll PR, Tlsty TD, Cunha GR. Carcinoma-Associated Fibroblasts Direct Tumor Progression of Initiated Human Prostatic Epithelium. Cancer Res (1999) 59(19):5002-11. doi: $10.1186 /$ bcr138

86. Kalluri R, Zeisberg M. Fibroblasts in Cancer. Nat Rev Cancer (2006) 6 (5):392-401. doi: 10.1038/nrc1877

87. Giannoni E, Bianchini F, Masieri L, Serni S, Torre E, Calorini L, et al. Reciprocal Activation of Prostate Cancer Cells and Cancer-Associated Fibroblasts Stimulates Epithelial-Mesenchymal Transition and Cancer Stemness. Cancer Res (2010) 70(17):6945-56. doi: 10.1158/00085472.CAN-10-0785 
88. Ippolito L, Morandi A, Taddei ML, Parri M, Comito G, Iscaro A, et al. Cancer-Associated Fibroblasts Promote Prostate Cancer Malignancy via Metabolic Rewiring and Mitochondrial Transfer. Oncogene (2019) 38 (27):5339-55. doi: 10.1038/s41388-019-0805-7

89. Cioni B, Nevedomskaya E, Melis M, van Burgsteden J, Stelloo S, Hodel E, et al. Loss of Androgen Receptor Signaling in Prostate Cancer-Associated Fibroblasts (Cafs) Promotes CCL2- and CXCL8-Mediated Cancer Cell Migration. Mol Oncol (2018) 12(8):1308-23. doi: 10.1002/1878-0261.12327

90. Henshall SM, Quinn DI, Lee CS, Head DR, Golovsky D, Brenner PC, et al. Altered Expression of Androgen Receptor in the Malignant Epithelium and Adjacent Stroma is Associated With Early Relapse in Prostate Cancer. Cancer Res (2001) 61(2):423-7.

91. Ricciardelli C, Choong CS, Buchanan G, Vivekanandan S, Neufing P, Stahl J, et al. Androgen Receptor Levels in Prostate Cancer Epithelial and Peritumoral Stromal Cells Identify non-Organ Confined Disease. Prostate (2005) 63(1):19-28. doi: 10.1002/pros.20154

92. Wikström P, Marusic J, Stattin P, Bergh A. Low Stroma Androgen Receptor Level in Normal and Tumor Prostate Tissue is Related to Poor Outcome in Prostate Cancer Patients. Prostate (2009) 69(8):799-809. doi: 10.1002/ pros.20927

93. Di Zazzo E, Galasso G, Giovannelli P, Di Donato M, Bilancio A, Perillo B, et al. Estrogen Receptors in Epithelial-Mesenchymal Transition of Prostate Cancer. Cancers (2019) 11(10):1418. doi: 10.3390/cancers11101418

94. Lin HK, Wang L, Hu YC, Altuwaijri S, Chang C. PhosphorylationDependent Ubiquitylation and Degradation of Androgen Receptor by Akt Require Mdm2 E3 Ligase. EMBO J (2002) 21(15):4037-48. doi: 10.1093/ emboj/cdf406

95. Keil KP, Abler LL, Laporta J, Altmann HM, Yang B, Jarrard DF, et al. Androgen Receptor DNA Methylation Regulates the Timing and Androgen Sensitivity of Mouse Prostate Ductal Development. Dev Biol (2014) 396 (2):237-45. doi: 10.1016/j.ydbio.2014.10.006

96. Lai KP, Yamashita S, Huang CK, Yeh S, Chang C. Loss of Stromal Androgen Receptor Leads to Suppressed Prostate Tumourigenesis via Modulation of Pro-Inflammatory Cytokines/Chemokines. EMBO Mol Med (2012) 4 (8):791-807. doi: 10.1002/emmm.201101140

97. Ricke EA, Williams K, Lee YF, Couto S, Wang Y, Hayward SW, et al. Androgen Hormone Action in Prostatic Carcinogenesis: Stromal Androgen Receptors Mediate Prostate Cancer Progression, Malignant Transformation and Metastasis. Carcinogenesis (2012) 33(7):1391-8. doi: 10.1093/carcin/ bgs 153

98. Montgomery RB, Mostaghel EA, Vessella R, Hess DL, Kalhorn TF, Higano CS, et al. Maintenance of Intratumoral Androgens in Metastatic Prostate
Cancer: A Mechanism for Castration-Resistant Tumor Growth. Cancer Res (2008) 68(11):4447-54. doi: 10.1158/0008-5472.CAN-08-0249

99. Nevedomskaya E, Baumgart SJ, Haendler B. Recent Advances in Prostate Cancer Treatment and Drug Discovery. Intern J Mol Sci (2018) 19(5):1359. doi: 10.3390/ijms19051359

100. Wu L, Yang Y, Guo X, Shu XO, Cai Q, Shu X, et al. An Integrative MultiOmics Analysis to Identify Candidate DNA Methylation Biomarkers Related to Prostate Cancer Risk. Nat Commun (2020) 11(1):3905. doi: 10.1038/ s41467-020-17673-9

101. Nguyen EV, Pereira BA, Lawrence MG, Ma X, Rebello RJ, Chan H, et al. Proteomic Profiling of Human Prostate Cancer-Associated Fibroblasts (CAF) Reveals LOXL2-dependent Regulation of the Tumor Microenvironment. Mole Cell Proteo: MCP (2019) 18(7):1410-27. doi: 10.1074/mcp.RA119.001496

102. Lawrence MG, Pidsley R, Niranjan B, Papargiris M, Pereira BA, Richards M, et al. Alterations in the Methylome of the Stromal Tumour Microenvironment Signal the Presence and Severity of Prostate Cancer. Clin Epigen (2020) 12 (1):48. doi: 10.1186/s13148-020-00836-2

103. Giovannelli P, Di Donato M, Galasso G, Monaco A, Licitra F, Perillo B, et al. Communication Between Cells: Exosomes as a Delivery System in Prostate Cancer. Cell Commun Signal: CCS (2021) 19(1):110. doi: 10.1186/s12964021-00792-1

Conflict of Interest: The authors declare that the research was conducted in the absence of any commercial or financial relationships that could be construed as a potential conflict of interest.

Publisher's Note: All claims expressed in this article are solely those of the authors and do not necessarily represent those of their affiliated organizations, or those of the publisher, the editors and the reviewers. Any product that may be evaluated in this article, or claim that may be made by its manufacturer, is not guaranteed or endorsed by the publisher.

Copyright (C) 2022 Licitra, Giovannelli, Di Donato, Monaco, Galasso, Migliaccio and Castoria. This is an open-access article distributed under the terms of the Creative Commons Attribution License (CC BY). The use, distribution or reproduction in other forums is permitted, provided the original author(s) and the copyright owner(s) are credited and that the original publication in this journal is cited, in accordance with accepted academic practice. No use, distribution or reproduction is permitted which does not comply with these terms. 